\title{
REMARQUES SUR LE GENUS COMMUNE DES NOMS DANS LA GRAMMAIRE LATINE
}

\author{
GUILLAUME BONNET \\ Université de Bourgogne (France)
}

\begin{abstract}
RÉSUMÉ: Les grammairiens latins distinguent d'abord trois genres nominaux, lesquels sont mal établis sur le plan référentiel, mais soutenus par l'évidence morphologique: masculin, féminin et neutre, ce dernier regroupant ce qui n'est, du point de vue sexuel, "ni l'un ni l'autre". Ils distinguent ensuite un genre commune, à comprendre initialement comme le genre de ce qui est "et l'un et l'autre". Ils réservent encore de la place pour un dernier genre, l'épicène grec, latinisé en promiscuum, lequel se distingue par le découplement entre genre du signifiant et sexe du signifié, de sorte à être mis à part des autres genera, lesquels renvoient, positivement ou in absentia, au sexus. On constate chez les grammairiens latins, pourtant, un flottement normatif entre les genres, lequel vient de l'hétérogénéité de la catégorie du genre chez les grammairiens latins, puisque commune et promiscuum ne sont pas, à proprement parler, des genres, étant dépourvus d'appui morphologique propre, mais plutôt des modes de référence, respectivement motivéee ou arbitraire, à la sphère extralinguistique.
\end{abstract}

MOTS-CLEFS: genres nominaux; commune et promiscuum "commun et épicène"; détermination morphologique et référentielle du genre.

Il est traditionnel, dans la littérature artigraphique latine de ne pas s'arrêter aux trois genres nominaux qui nous sont restés: masculin, féminin et neutre. L'expression linguistique n'étant pas détachée du monde des référents, la considération du sexe interférait avec les catégories linguistiques; c'est ainsi déjà qu'aux masculin et féminin, évidents, avait été ajouté un neutrum, regroupant ce qui n'est, du point de vue sexuel, "ni l'un ni l'autre". Cette liste des genres "principaux" (cf. Diom., GL I 301, 4) est mal établie sur le plan référentiel (pourquoi mancipium, désignant les esclaves, est-il neutre?), mais soutenue par l'évidence morphologique: les 
noms "mobiles" en genre (nos adjectifs) ont les trois, et la catégorie du neutre reçoit même de la deuxième classe des adjectifs une justification formelle: forte s'oppose à fortis masculin et féminin. Apparaît ensuite, selon un processus qui trouve, de par son efficacité pédagogique, un parallèle dans les voix verbales (cf. Flobert 1975, 11), un genre commune, à comprendre initialement comme le genre de ce qui est et l'un et l'autre. Ce genre sexuellement ambivalent (canis "chien" et "chienne") connaît à son tour une subdivision visant à mettre en valeur le (sous-)genre omne, concernant les mots pouvant endosser les trois catégories "primaires", masculin, féminin et neutre ${ }^{1}$, tels felix. A côté de ce système raffiné, il reste encore de la place pour un dernier genre, l'épicène grec, latinisé en promiscuum, dont la dernière place est assortie d'un statut spécial: c'est que la catégorie se distingue par de découplement entre genre du signifiant et sexe du signifié. Cet arbitraire fait que le genus promiscuum est mis à part des autres genera, lesquels renvoient positivement ou in absentia, au sexus.

Ce maillage théorique serré, quoique non homogène, peut laisser penser que tous les substantifs, surtout les plus fréquents, y trouvent une étiquette identique d'une grammaire à l'autre ${ }^{2}$. Cependant, malgré la pratique des Artes grammaticae qui imitent le grec en recourant à hic haec hoc, le latin ne bénéficie pas de l'“immédiateté" du genre propre aux langues qui ont un article différencié en genre à associer aux noms. La détermination générique est alors plus vague: morphologique - par le recours à une comparaison que viennent toujours contester des exceptions - ou référentielle, elle est parfois, faute de mieux, aléatoire ${ }^{3}$ : Priscien peut ainsi exposer les deux genres attestés de silex en opposant Juvénal et Ovide à Virgile (GL II 160, 13-19) sans argument pour l'un plus que pour l'autre: nos dictionnaires modernes répercute cette hésitation. Si pour certains mots la rareté est cause d'une hésitation qu'on peut d'autant mieux comprendre qu'il y a un décalage entre la langue littéraire, objet du commentaire érudit, et la pratique contemporaine des élèves, pour d'autres, cependant, qui relèvent de la langue quotidienne, la divergence des points de vue est plus surprenante. Nous voudrions examiner dans les pages qui suivent le cas des substantifs revendiquant le genre commune.

\footnotetext{
Sur les hésitations concernant commune et omne, cf. Bonnet 2008, 183-185.

2 Ainsi de bos par exemple, commune par héritage i.e., ce que ne contestent pas les grammairiens (sauf, par inadvertance, Probus Palladius, Instituta artium 120, 5 (cf. ibid., 121, 37 sqq.).

3 Face à ce qui paraît donc arbitraire, on ne peut qu'énumérer: ainsi Donat, Mai. 620, 1-5 $\mathrm{H}$, avec des noms sono masculina, mais intellectu feminina, sono feminina mais intellectu masculina, etc.
} 


\section{Hic homo: et haec?}

Le point de départ de notre réflexion sera un passage de l'Ars breuiata attribué à saint Augustin, dont nous préparons la réédition sur de nouveaux frais. Dans le $\$ 5$ de l'édition Weber (1861), qui traite de l'accident nominal du genre, le mot homo est donné comme l'exemple type du commune utriusque sexus. Les lecteurs français ou portugais pourront tout d'abord s'étonner, puisque dans ces deux langues romanes, la continuation phonétique du mot latin (donné comme masculin dans le Gaffiot), pg. om, frs on et homme, est régulièrement du genre masculin. Mais de fait, la morphologie des mots latins en *-ōn est indécise, donnant des masculins (les sobriquets) ou des féminins (les séries en -io, -tio, -tudo), et à bien y regarder toutefois, on se souviendra du passage de Juvénal (VI 284), où une femme revendiquant sa liberté, déclare "homo sum". Enfin, il y a le témoignage péremptoire de grammairiens qui allèguent le genre commune de homo. Chronologiquement, ce sont Diomède (301, 9-104), l'autre Pseudo-Augustin, le rédacteur des Regulae (502, $4^{5}$ et 513, 10-116), l'auteur anonyme de la grammaire de Bobbio (20,30 De Nonno), et particulièrement Priscien, qui revient à cinq reprises sur le mot. Si trois occurrences consistent en de simples mentions (GL II 142, 25; 284, 5; Institutio de nomine 13, 10 P), une quatrième est plus significative $(146,3-9)$ :

Alia uero omnia [scil.nomina in -o desinentia] masculina sunt, ut stellio, Anio, Cicero, Milo, Apollo, mango, praedo, ligo, carbo, sermo, quaternio, exceptis paucis communibus, quae communia esse tam ipsa natura quam exempla Graecorum nobis demonstrare possunt, apud quos uel communia uel mobilia haec inueniuntur, ut hic et haec homo, ó

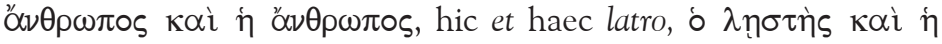
$\lambda \eta\rceil \sigma \eta \dot{s}$.

On voit clairement ici le jeu entre déterminations morphologique et référentielle. C'est bien sous l'effet de leur référent que ces mots sont communia: le grec, qui place couramment l'article féminin devant les équivalents de latro et homo est à cet égard très explicite; mais il est présenté surtout comme le modèle. De fait, on peut se demander s'il n'y a pas là une extension imitative du genre au féminin

4 Sunt enim communia duum generum ex masculino et feminino, ut hic et haec homo...

5 A la première place d'une liste de mots en -o (homo, ganeo, degulo, caupo, fullo, nemo, helluo, glutto "et talia") donnés comme communia, et dont le modèle, latro, est décliné dans les lignes précédentes. Nous retrouverons ce passage plus loin.

6 En parallèle explicite à l'occasion de la présentation des verbes communia. 
pour deux mots qui sont, dans l'immense majorité de leurs emplois, utilisés pour renvoyer à des êtres masculins. Mais Priscien ne s'en tient pas là, et propose, plus loin (GL II 206, 8 sqq.), une série de citations à l'appui de la même affirmation, le genre commune de homo:

quae in genetiuo non seruant o [...] homo hominis, quod est commune. Cicero pro Cluentio [70, 199] cum de eius matre loqueretur, ait: "cuius ea stultitia est, ut eam nemo hominem appellare possit", et Lucanus in $\mathrm{V}$ [168-169] de Phemonoe: "atque hominem toto sibi cedere iussit / pectore". Virgilius in I [328]: "nec uox hominem sonat", de femina loquens.

On voit que l'argument avancé par Priscien est le sexe féminin du référent extratextuel: la redoutable mère de Cluentius, la pythie Phémonoe en pleine possession, Vénus enfin, qui vient de s'adresser à Enée. Pourtant, l'analyse moderne n'est pas convaincue par la pertinence de ces exemples. Si homo a le genre féminin, il devrait signifier "femme"; or, son sens dans les différents exemples ne traduit pas une évolution de "homme" / uir à "femme"; il dépasse plutôt toute référence sexuée, pour renvoyer à ce qu'il y a, ou devrait y avoir, de proprement humain dans l'une ou l'autre des créatures - ici féminines - considérées: c'est ainsi que Virgile peut écrire, en En. III 426, que le monstre Scylla exhibe une "hominis facies"! Enfin, un indice aurait pu détourner Priscien de cette interprétation: si l'on élargit la citation de Cicéron, on constate que s'y construit une brillante gradation de homo à femina, puis à mater, ce qui montre bien qu'il n'y donne pas à homo le sens de "femme"!

Ces lectures de Priscien sont-elles partagées? Si les commentaires antiques de Lucain dont nous disposons sont ici muets, les médiévales Glosule super Lucanum d'Arnulf d'Orléans (éd. Berthe M. Marti, American Academy in Rome, 1958) comprennent "hominem: humanitatem". Du passage de Virgile, Tiberius Donatus reformule la réponse d'Enée à sa mère (Interpretationes uergilianae, p. 69, 25-27): quae, inquit, in te uideo uel cum loqueris auribus sumo non te humanae condicionis adsignant. Autrement dit, il comprend bien qu'on a ici une métonymie: homo pour humana condicio! Le commentaire de Servius est, quant à lui, laconique et passablement énigmatique: "Graeca figura est", dit-il. Nous serions tenté de comprendre qu'ici, le commentateur entend bien homo comme féminin, mais selon une licence inspirée

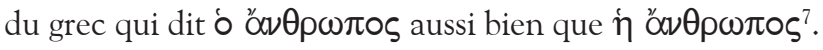

7 Pour ce vers, les commentateurs modernes évoquent tous l'accusatif d'objet interne; est-ce ce à une extension de l'“accusatif grec" que renverrait le jugement de Servius, 
Dans tous ces emplois ${ }^{8}$, homo désigne en tout cas l'ensemble des caractéristiques propres à l'homme et à la femme, par opposition éventuellement à un dieu (Vénus). Il s'agit d'un hyperonyme, un nomen generale, et non plus d'un exemple type de nomina specialia, catégorie qu'il illustre souvent (cf. Donat, Mai. 617, $5 \mathrm{H}$ ). Se pourrait-il que Priscien ne ce fût pas aperçu du changement de sens? Un dernier passage grammatical posant le genre commune de homo, passage qui nous a été conservé par Charisius - dont on sait que l'Ars compilatoire puisait à des sources anciennes ${ }^{9}$ - permet d'entrevoir une position théorique qui expliquerait la position curieusement insistante de Priscien sur ce point (45, 5-8 B):

quae in o terminantur masculini generis, si tamen recipiant et femininam naturam, erunt communia, uelut hic et haec latro, nebulo E'ik $\alpha i$ os, homo et similia, exceptis his, leno lena, leo lea, et siqua alia.

Le grammairien anonyme pose ici un dédoublement dénotatif des mots en questions: quand ils admettent aussi la natura féminine, ils deviennent communia, ce qui veut dire qu'en des contextes référentiels moins complexes, ils ne cessent pas d'être masculins. Le dédoublement morphologique qui s'ensuit est finalement légitimé par la polysémie de homo, qu'on pourrait formuler en terme d'analyse antique en disant que ce nomen est tantôt speciale (par rapport à equus, et subsumé par animal, selon le découpage traditionnel, mais aussi face à mulier/femina), tantôt generale (particulièrement en poésie, par rapport à superi, mais aussi face au couple uirfemina: cf. Diom. 320, 18-22). La traduction de son statut de nomen generale, c'est alors le genus commune.

Ce distinguo, reflété par Diomède, l'anonyme de Bobbio, l'auteur des Regulae, et Priscien, n'a pas été autrement retenu, comme le montre la fréquence des mentions non commentées de homo nom masculin - par exemple, comme source de diminutifs eux-mêmes masculins, alors qu'un changement de genre eût appelé immanquablement une remarque: cf. Dos. 21, 14-16 B. Ignoré le plus souvent, il est

alors que ce tour précis est attesté en latin, par ex. Off. III, 21? Sa glose de Ec. I 5 (pour resonare Amaryllida), semblable pour la construction, ne permet pas de le supposer.

8 Et d'autres, qu'on pourra trouver ici ou là; ainsi, de la Correspondance de Cicéron (Ep. 4, 5, 4 = 597 CUF) "homo nata fuerat" écrit de Tullia Ser. Sulpicius Rufus cherchant à consoler Cicéron en mars 45.

9 Cominien et Julius Romanus au moins. Sur la difficile question des sources de Charisius, on se reportera en dernier lieu à P. L. Schmidt, Restauration und Erneuerung. Die lateinische Literatur von 284 bis 347 n. Chr., München, 1989 (vol. 5 du nouveau "Schanz-Hosius"). 
contesté implicitement par le simple rappel du genre masculin du mot (cf. Phocas, De nom. et uerbo 33, 15 C). Quant à la possibilité qu'on ait affaire à un épicène, elle n'est même pas envisagée...

Nous devons au caractère parfois indistinct de la compilation charisienne une ferme critique, explicite, de l'extension du genre, dans un passage qu'on pourra supposer avec une certaine vraisemblance tiré de Julius Romanus (130, 19 sqq. B):

heres, parens, homo etsi in communi sexu intellegantur, tamen masculino genere semper dicuntur. Nemo enim aut secundam heredem dicit aut bonam parentem aut malam hominem, sed masculine, tametsi de femina sermo habeatur. Nam Marcus ait "heredes ipsus secundus", et Pacunius in Medo [219 R3], cum ostenderet a Medo matrem quaeri, ait "te, Sol, inuoco ut mihi potestatem duis inquirendi mei parentis". Sed Gracchus "suos parentes amat" cum dicit in significatione matris * et in alia epistula "tuus parens sum" ait, cum de se loqueretur. Et apud Vergilium sic legimus [En. III 341] "ecqua tamen puero est amissae cura parentis".

Relevons la fermeté de l'opposition intellegantur / dicuntur, qui distingue le plan de la référence, et celui de la langue. L'usage est ensuite convoqué pour prouver l'absurdité "à l'oreille" de la position soutenant le genre commune de ces mots. Suivent enfin des auctoritates, dans une dernière partie du texte malheureusement détériorée. Les plus anciens auteurs, dont l'exemple pèse le plus lourd en termes d'authenticité, soutiennent le genre masculin. On peut ensuite deviner, pour le seul mot parens toutes les citations intéressant homo ont disparu, et heres n'est alors plus illustré - un contre-exemple tiré d'une lettre de Cornélie, mère des Gracques (?), et un autre de Virgile ${ }^{10}$. Cette défense du caractère finalement épicène de homo, parens et heres quitte donc le terrain théorique pour se placer sur le terrain syntagmatique: une association du genre féminin avec ces mots est présentée comme inenvisageable, sauf licentia auctoritatum. Que disent les textes?

\section{Plan référentiel, plan syntagmatique}

Pour parens, la situation de départ est complexe: du participe de pario, c'est le féminin qu'on attendrait au singulier, tandis que le pluriel devrait être masculin,

10 Que l'auteur n'ait pas songé au célébrissime "hoc erat alma parens" (En. II 664; exemple de syllabe longue par position: al-ma) qui hante tous les manuels tardifs semble confirmer l'antiquité de cette source. 
compte tenu de la précellence du masculin dans des associations hétérogènes. Une telle disparité était vouée à l'uniformisation. La plus grande fréquence des occurrences au pluriel a dû imposer le masculin au singulier, en dépit d'un référent éventuellement féminin: c'est ce dont témoigne l'extrait de Pacuvius, et peut-être le "tuus parens sum", s'il faut attribuer ce fragment à Cornélie ${ }^{11}$. Les emplois virgiliens, qui réactivent dans parens le sens de pario, constitueraient alors une figure, syllepsis per genera. En termes grammaticaux "tardifs", on attendrait donc une analyse comme mot épicène, réserves faites des emplois virgiliens. C'est bien ce que paraît confirmer une differentia ${ }^{12}$ :

inter patrem et parentem: pater est alicuius...*..., parens uel masculus uel femina. Quod inter coniux et uirum interest, hoc inter parentem et patrem: coniux uterque dicitur, uir qui maritus est. Ita et parens uterque, alter quod sit causa partus, alter quod pariendi habeat materiam.

Le genus commune, alternative au promiscuum que semble pointer cette differentia ${ }^{13}$, est cependant défendu par le Pseudo-Marius Victorinus / Palémon (191, 18 et 20) et surtout Priscien - encore lui - (GL II 319, 18 et 354, 24), tous deux sans doute impressionnés par la pratique poétique, virgilienne et postérieure. Au reste, le vocatif associé à un adjectif féminin dans l'adresse à une entité divine et abstraite, sur le modèle de En. II 664 ou Georg. II 173, devient une marque de grand style, enseignée comme telle par les professeurs comme Térentianus (cf. 29, 342 et 101, 1390 Cignolo) ou Fortunatianus (GL VI 288, 1).

Le genre de heres pose un problème différent: il ne dépend pas de la polysémie, comme homo, ni ne se complique des hasards de l'étymologie, comme parens. Il ne s'agit plus ici que de référence extra-linguistique pour un terme en usage dans la langue technique du droit. Le témoignage recueilli par Charisius pose donc le genre masculin même si le référent est féminin, ce qui en fait un épicène. Lavis de Priscien (GL II 243, 8 et Institutio de nomine 15, 7 P), comme celui de Phocas (De nomine et uerbo 39, 10 C) est qu'on a là un mot du genus commune. Les deux

11 On rappellera en outre Festus (137.16 L) "Masculino genere parentem appellabant antiqui etiam matrem".

12 Coll. dite "de Palaemon", supposée extraite des Prata de Suétone, dans l'édition Roth de Suétone, Leipzig, 1858, p. 309.

13 On notera que c'est de pater qu'on cherche à distinguer parens, dont le sexe du référent, non le genre, est évoqué par les mots masculus et femina. 
grammairiens tirent les conclusions d'une analyse syntagmatique, et non syntaxique, des emplois juridiques où le mot heres est associé à un adjectif féminin. L'examen d'un certain nombre de cas, relevés dans les codes, où heres désigne une femme, conduit à isoler ceux où l'on a affaire à un mot employé seul comme attribut ${ }^{14} \mathrm{ou}$ apposition $^{15}$ : aucun indice ne marque le genre, et l'on interprétera donc ces emplois avec la source de Charisius comme épicènes. Restent les constructions où heres est accompagné d'une épithète au féminin, dont l'accord indiquerait qu'on a alors affaire à un genus commune. On ne rencontre guère, dans ce cas de figure, que ${ }^{16}$ l'adjectif indéfini sola, à deux reprises: a) Iulianus, Dig. 23, 4, 22: filiam suam solam heredem reliquerat; b) Scaeuola, Dig. 29, 7, 98: in Seiam quae sola heres remansit..., ut sola heres erat.

Il apparait dans la langue juridique une locution "sola heres", correspondant à une situation juridique particulière. Nous sommes tentés de voir là le point de départ d'un emploi effectif comme féminin de heres, qu'on retrouve ici ou là, et d'abord dans une langue encore technique: la Controverse 2, 7 du recueil de Sénèque le Rhéteur ("sola heres esto" trois fois, dans les $§ 6$ et 7) ${ }^{17}$. La même Controverse, dans son argument, fait apparaittre un syntagme heredem formosam avec un accord au féminin qui entérine le passage du mot du genre masculin au genre commune. Plus tard, on trouve chez Jérôme, Chron ab Abr. a. 2054 [= 41 av. J.-C.], le même accord: Passienus filius fraude heredis suae necatus ${ }^{18}$.

14 Cic. Verr. III, 21, 41: "heres erat filia”; Pomponius, Dig. 29, 2, 99: "cum duae filiae patri necessariae heredes exstitissent...”; Gaius, Inst. 3, 42 "cum unum filium unamue filiam heredem reliquerit libertus... cum uero duos duasue heredes reliquerit..."; Scaeuola, Dig. 50, 17, 73: "quo tutela redit, eo et hereditas peruenit, nisi cum feminae heredes intercedunt". On ajoutera les occurrences où figure sua, "capable juridiquement” le plus souvent immédiatement préposé à heres, qui fonctionne cependant comme apposition au support de l'attribut, et non comme épithète (Gaius Inst. 3, 3: "uxor... quae in manu uiri est, ei sua heres est quia filiae loco est"; Tryphoninus, Dig. 37, 7, 9: "si sua heres filia patri cum fratribus contenta dote abstineat se bonis..."; Cod. Iust. 6, 55, 8 (a. 294): "patre defuncto filia... heres extitit sua"), ainsi qu'un passage où l'adjectif nous semble devoir être analysé de la même manière: Gaius, Inst; 3, 14: "soror fratri sororiue legitima heres, amita uero et fratris filia legitima heres esse non potest". Le passage de Gaius, Inst. 3, 40: "filius filiaue uel uxor quae in manu esset suus uel sua heres esset...", avec les deux sujets, montre l'attribut épicène en situation.

15 Scaeuola, Dig. 32, 42: ab utraque herede.

16 Sous réserve de l'analyse de legitima de l'exemple cité dans la note précédente.

17 En plus de trois emplois comme attribut du sujet féminin, aux $\S 8$ et 9.

18 Mais le passage: trade comitem sanctitatis, futuram heredem, de sa Lettre 107, 13, 3 nous livre un attribut renvoyant certes à une femme (la petite-fille d'Eustochie) mais sans 


\section{Genus commune et genus "subcommune"}

Avec l'autre substantif donné traditionnellement comme exemple du genus commune, sacerdos ${ }^{19}$, on voit bien que la référence extralinguistique force le genre féminin d'autant plus aisément qu'il s'agit d'un nom de fonction ${ }^{20}$. Mais la précision syntagmatique éventuelle, moyennant une épithète, n'est pas jugée suffisante, et l'apparition épisodique de féminins alternatifs, sacerdota, sacerdotissa (cf. Ernout/ Meillet: sacerdos) traduit une volonté de sortir décidément du genus commune selon un procédé classique de suffixation du féminin.

A moindres frais sur le plan lexical, et toujours faute d'un marquage morphologique individualisant le féminin, tous les noms épicènes de fonctions ou de métiers employés comme apposition ou attribut de support féminins tendent à être interprétés sur le coup comme féminins, ce qui entraîne le changement de catégorie générique du substantif, du masculin épicène au genus commune. Tel est le cas de custos, sous l'influence d'Horace, Carm. III 22, 1, (Probus-Palladius, Instituta artium 92, 20; Priscien, GL II 161, 23, Phocas De nom. et uerbo 41, 15 C); de augur (Priscien, GL II 155, 2-3; Phocas, De nom. et uerbo 38, 8 C) d'après le même Horace (Carm. III 17, 12-13)...

Que les auctoritates la cautionnent ou non - mais les grammairiens ne donnent pas toutes leurs sources, dont une partie s'est perdue -, on constate une tendance à l'élimination des mots épicènes au profit d'un classement dans le genre commune: ainsi Probus-Palladius, Instituta artium, 93, 13-15 établit-il une liste des communia classés par types de syllabe finale, où nous découvrons avec surprise tibicen ${ }^{21}$, optimas, uates, sacerdos, artifex, dux, caelebs, princeps, coniunx. De listes semblables figurant chez Priscien (GL II 142, 24 sqq. ou 284, 4-6), nous tirons en outre aduena, ciuis et symptomatiquement auctor (cf. En. XII 159, dit de Junon), donc par extension tous les noms en -tor dépourvus d'un dérivé féminin en -trix. Quelques réactions à cette tendance sont observables, (ré) affirmant le genre masculin, donc le caractère épicène, de tel ou tel mot: ainsi dans les Catholica du Pseudo-Probus, 33, 3, pour sacerdos. Charisius $(114,23-26$ B) cite une source anonyme refusant explicitement le genus commune pour sacerdos et custos (alors qu'en 116, 17-18 B, custos est traité comme du genre omne!).

accord proprement dit.

19 Donat, Min. 586, 6 H etc., ainsi que ses commentateurs Servius, Pompée, Clédonius; les Regulae, 502, 19 sqq.; Phocas, De nom. et uerbo 41, 16 C; Priscien, GL II 141, 12 et alibi

20 Déjà chez Plaute, Rud. 329: "sacerdos Veneria” à côté de ibid. 433 "sacerdos Veneris".

21 Malgré l'existence de tibicina chez Térence [Eun. 457], rappelée par Sacerdos 474, 14 ! 
Ces hésitations traduisent la difficulté à distinguer communs et épicènes en l'absence de l'immédiateté du genre que donnent l'article du grec - en l'absence aussi d'outils grammaticaux distinguant l'adjectif, épithète, et le substantif, apposé. Des artigraphes se sont efforcés de poser tout au moins le problème, sinon de le clarifier. Pompée (161, 4-31) et l'anonyme auteur des Explanationes in Artes Donati (494, 1 sqq.) reprennent pour leur compte et adaptent une explication qui pose en principe la distinction entre les deux genres dans la possibilité d'identifier le sexe du référent: ubi uisu discernimus sexum. Le genre épicène fait alors figure de genre "d'attente" pour des signifiants potentiellement communia, s'ils viennent à bénéficier d'une plus grande précision ${ }^{22}$. Quant à l'arbitraire originel inhérent aux mots décidément épicènes, il est parfois modifié par l'initiative plus ou moins expliquable d'auctoritates: in miluo legimus enim et masculino genere, legimus et feminino: rapidissima miluus exit [Ovide, Mét. II 716], écrit l'anonyme (494, 26-27). C'est très exactement décrire le mouvement de soutirage qui vide la catégorie des épicènes au profit de genres contextuellement motivés, selon les nécessités pédagogiques dictées par les commentaires scolaires.

La tentation de l'épuisement de la question par l'énumération se fait jour dans les Regulae du Pseudo-Augustin, dont le propos est, il est vrai, strictement normatif. On trouve, au fil de la section reprenant les noms classés par la finale (501, 32 - 504, 3 - nous ôtons les adjectifs), aduena Troiugena, indigena, incola, agricola, conuena, caelicola, sacricola, publicola, terricola, urbicola, plebicola, Numida, Dalmata, ruricola, lucifuga, Persa, Romulida, latro, homo, ganeo, degulo, caupo, fullo, nemo, helluo, glutto, comes, sospes locuples, hospes, miles, ueles, interpres, ales, praes "fideiiussor", satelles, deses, praeses, Caeres, caeles, indiges, ciuis, testis, hostis, sacerdos, custos, nepos, sus, opifex, artifex, aurifex, iudex, carnifex, index, uindex, auspex, haruspex, signifex, princeps, manceps, particeps, municeps, coniux, dux, redux, celebs, Phryx, Ligus, pugil, praesul, fur, augur, auctor!

Ce mouvement de motivation sexuelle du genre des noms va dans la même direction que les phénomènes de développement morphologique bien connus aboutissant, pour des adjectifs de la première classe ou des substantifs féminins en $-a$ correspondant à des masculins thématiques, à l'apparition de finales en -abus

22 C'est ainsi que nous interpréterions volontiers la désignation, unique dans la tradition latine, du genre épicène par le terme subcommune (Diom. 301, 13): "un peu, presque commune" (cf. subiratus "demi fâché"). La fabrication de cet hapax comme un calque de gr. '̇ं'́k Kolvov, proposée par Job 1893, 62 et admise par Shad 2007, 381 ne nous convainc pas. 
par exemple. C'est la même nécessité de préciser le sexe, dans un cadre juridique par exemple, qui a créé un filiabus à opposer à filiis, alors seulement masculin, et pousse Jérôme (cf. supra) à écrire heredis suae pour renforcer l'horreur du crime ayant une femme comme auteur. Les grammairiens accueillent avec une certaine jubilation ces formes extraordinaires collectées ici ou là ${ }^{23}$. Et il est remarquable que les deux développements sur la confusion communia / promiscua dont nous venons de parler juxtaposent un miluus exceptionnellement féminin d'Ovide [Mét. II 716] et le métaplasme turdarum, créé par Perse (VI 24), confondant significativement genre et marquage morphologique.

Le flottement normatif entre genera masculinum, commune et promiscuum vient de l'hétérogénéité de la catégorie du genre chez les grammairiens latins, et cette hétérogénéité est due essentiellement à la manie taxinomique des artigraphes qui, pour des raisons pédagogiques sans doute, divisent à l'infini les catégories en groupes et sous-groupes de comportement homogène. Commune et promiscuum ne sont pas, à proprement parler, des genres, puisqu'ils sont dépourvus d'appui morphologique propre, mais plutôt des modes de référence, respectivement motivée ou arbitraire, à la sphère extra-linguistique. Il est aisé de retrouver, dans les errements provoqués par leur assimilation aux “vrais" genres, l'incapacité de la grammaire antique à constituer le langage comme un objet de discours en soi, hors de toute référence à la réalité de ce qui est dit; mais au-delà du discours forcé24, il y a une réalité entrainante à prendre en compte: la correspondance spontanée que les locuteurs établissent entre genre du signifiant et sexe du signifié: on la voit à l'œuvre avec heres formosa ou sacerdota, avec libertabus, etc. On retrouve ce trait "populaire" dans les langues modernes; qu'il suffise de citer ici frs collègue, dont le genus commune s'est imposé au seul masculin à la faveur de la féminisation du monde du travail.

Mais retournons à nos grammairiens. L'Antiquité tardive est l'époque de la christianisation de la pensée occidentale. Il n'est donc pas étonnant de voir la grammaire, précisément dans le flou qui entoure genera commune et promiscuum,

23 On trouvera signalés chez les grammairiens asinabus, deabus, equabus, filiabus, iustabus, libertabus, magnabus, mimabus, mulabus, natabus, oleabus, paucabus, pudicabus, puellabus, raptabus, uerabus.

24 Lanonyme auteur carolingien de l'Ars Bernensis, qui a lu Priscien, regimbe ainsi contre les conséquences de ce qu'il consigne à la suite de son maître: l'élimination du genre épicène au profit du genus commune. Il accepte bien (GL VIII 110, 7 et 9) haec homo, mais refuse hanc hominem. Le genre commune s'est donc imposé théoriquement, mais la pratique en reste à un mot épicène! 
sollicitée par cette tâche. Ainsi de homo, particulièrement sensible dans le discours théologique. Lindistinction référentielle du genre épicène est providentielle aux yeux du rédacteur d'une curieuse notice du De dubiis nominibus (580, $2=779$, 9-10 Glorie): homo promiscui generis; nam ut uir "homo", ita et mulier "homo" dicitur; et Iesus ideo "filius hominis", quia filius uirginis. L'équivalence homo - uirgo vise à défendre la perfection de l'humanité (c'est le sens de "Fils de l'homme") de Jésus, "vrai Dieu et vrai homme", qui ne pouvait pourtant revendiquer un père humain; et cette équivalence se déduit du genre épicène, qui affecte indivisiblement masculin ou féminin, uir ou uirgo. En s'appuyant sur la position inverse, le Pseudo-Augustin de l'Ars breuiata $(\S 5 \mathrm{~W})$ introduit homo comme exemple de nomen commune, nous l'avons vu plus haut, et exploite l'opposition avec les deux exemples précédemment donnés par lui de masculin et de féminin, fortement sexués, uir et mulier. Ce grammairien chrétien - on le voit à d'autres traits de son Ars - fait implicitement référence à Gn I, 27: et creauit Deus hominem ad imaginem suam; ad imaginem Dei creauit illum; masculum et feminam creauit eos. L'humanité, représentée par Adam et Eve, veut un substantif commune, apte à référer pleinement (jusque dans la "vérité" de l'accord grammatical) et de manière suffisante aussi bien à l'homme qu'à la femme...

Les querelles de grammairiens ont des prolongements insoupçonnés.

\section{RÉFÉRENCES BIBLIOGRAPHIQUES}

Les auteurs anciens sont cités d'après les éditions courantes. On n'a indiqué la tomaison des Grammatici Latini que pour Priscien.

Bonnet, G. "La forme des Artes grammaticae, reflets de la pratique des maîtres", dans

Aspetti della scuola nel mondo romano, Atti del Convegno (Pisa, 5-6 dicembre 2006) a cura di Franco Bellandi e Rolando Ferri, Amsterdam, Adolf M. Hakkert, 2008, 179-188.

Flobert, P. Les verbes déponents latins, des Origines à Charlemagne, Paris, Les Belles Lettres, 1975.

JoB, L. De grammaticis vocabulis apud latinos, Lutetiae Parisiorum, Bouillon, 1893 (diverses réimpressions, 2010)

ScHAD, S. A lexicon of Latin grammatical terminology, Pisa - Roma, Fabrizio Serra, 2007. 
BONNET, G. Notas sobre o genus commune dos nomes na gramática latina.

RESUMO: Os gramáticos latinos distinguem, primeiramente, três gêneros nominais, que se apóiam imperfeitamente no plano referencial, mas são sustentados pela evidência morfológica, a saber: o masculino, o feminino e o neutro, o qual recobre o que não é, do ponto de vista do sexo, "nem um nem o outro". Daí, distinguem um gênero commune, entendido primeiramente como o gênero do que é "tanto um como o outro". Enfim, reservam lugar a um último gênero, o epiceno grego, dito em latim promiscuum, que se distingue por dissociar o gênero do significante do sexo do significado, sendo por isso posto à margem dos outros genera, que remetem, positivamente ou in absentia, ao sexus. Constata-se nos gramáticos latinos, porém, uma flutuação normativa dos gêneros, a qual se deve à heterogeneidade da categoria do gênero praticada por aqueles gramáticos, uma vez que commune e promiscuum não são propriamente gêneros, já que desprovidos de apoio morfológico próprio, mas antes modos de referência, respectivamente motivada e arbitrária, à esfera extralingüística.

PALAVRAS-CHAVE: gêneros nominais; commune e promiscuum "comun e epiceno"; determinação morfológica e referencial do gênero. 